DOCUMENTS

pour I'histoire
des techniques
Documents pour l'histoire des techniques

Nouvelle série

$17 \mid 1^{\text {er }}$ semestre 2009

L'invention technique et les figures de l'inventeur (XVIII $-X^{\mathrm{e}}$ siècles)

\title{
Les autorités coloniales françaises et l'économie artisanale à Maroua (Cameroun)Bilan historiographique
}

French colonial authorities and handicraft economy in Maroua (Cameroun). A historiographic review

\section{François Wassouni}

\section{OpenEdition}

Journals

Édition électronique

URL : http://journals.openedition.org/dht/547

DOI : $10.4000 /$ dht. 547

ISSN : 1775-4194

Éditeur :

Centre d'histoire des techniques et de l'environnement du Cnam (CDHTE-Cnam), Société des élèves du CDHTE-Cnam

\section{Édition imprimée}

Date de publication : 31 mars 2009

Pagination : 149-161

ISBN : 978-2-95-30779-3-3

ISSN : 0417-8726

\section{Référence électronique}

François Wassouni, « Les autorités coloniales françaises et l'économie artisanale à Maroua (Cameroun)Bilan historiographique », Documents pour I'histoire des techniques [En ligne], 17| 1 $^{\mathrm{er}}$ semestre 2009, mis en ligne le 28 septembre 2010, consulté le 07 septembre 2020. URL : http:// journals.openedition.org/dht/547 ; DOI : https://doi.org/10.4000/dht.547 


\title{
Les autorités coloniales françaises
}

et l'économie artisanale

à Maroua (Cameroun).

Bilan historiographique

\author{
François Wassouni * \\ Université de Ngaoundéré
}

\begin{abstract}
RÉSUMÉ
Le présent travail analyse l'apport des autorités coloniales françaises dans l'artisanat de Maroua, chef-lieu de la région de l'Extrême-Nord du Cameroun. Développé au XIXe siècle par les communautés kanouri et haoussa, l'artisanat de Maroua comprend le cuir, les textiles traditionnels, la forge, la vannerie. Très tôt, cet artisanat fascine les colons français qui s'engagent à le « moderniser » en s'investissant dans l'amélioration des techniques de production et des moyens de commercialisation. Diverses actions entraînent le développement de l'artisanat qui, aujourd'hui encore, occupe de nombreuses personnes. Cependant, les objets fabriqués sont inspirés de la civilisation occidentale et s'adressent à une clientèle européenne. Pour autant, les autorités coloniales auront contribué à donner une impulsion à ce secteur d'activité qui reste l'une des spécificités de la ville de Maroua.
\end{abstract}

Résumés et mots clés en anglais sont regroupés en fin de volume, accompagnés des mots clés français

\begin{abstract}
'Extrême-Nord est une région du Cameroun qui Lregorge de potentialités touristiques, telles que le Parc National de Waza, le Pic de Mindif, le Parc de la Kalamaloué et la région de Rhumsiki. A cet attrait naturel de la région, s'ajoute l'artisanat, riche d'une mosaïque de produits qui fascinent et attirent les visiteurs. II en est ainsi de la ville de Maroua. Située dans la partie septentrionale du Cameroun à 10²' de latitude Nord et $14^{\circ} 4^{\prime}$ de longitude Est, Maroua est le chef-lieu de la région de l'Extrême-Nord et du département du Diamarél. Au rang des activités de cette cité caractérisée par l'interaction entre les activités économiques traditionnelles et modernes, figure donc l'artisanat. Ce secteur occupe une place de choix dans la ville, avec des filières telles que le tissage traditionnel, la forge, la vannerie, la teinturerie
\end{abstract}

1 BCEOM, Ville de Maroua. Plan d'Urbanisme Directeur Horizon 2000, 1981, p. 3. et surtout le travail du cuir qui lui donnent une certaine spécificité ${ }^{2}$. Le Centre artisanal de cette ville offre la possibilité aux visiteurs de découvrir la production; il est le lieu par excellence d'achat et de vente des produits locaux.

L'artisanat s'est développé à Maroua au XIX siècle grâce aux communautés kanouri et haoussa. Mais c'est surtout au XXe siècle, précisément entre 1930 et 1955 que ce secteur a pris de l'importance avec la présence des colonisateurs français. Ayant constaté la richesse de cet art local, les Français se sont engagés à le promouvoir en y apportant de nombreuses innovations.

De nombreux auteurs ont consacré des travaux sur la ville de Maroua, lesquels travaux font mention à différents niveaux de l'artisanat. L'on peut citer

2 Christian Seignobos et Olivier lyébi-Mandjek, Atlas de la province de l'Extrême-Nord, Paris, IRD, 2000, p. 160.

\footnotetext{
* Doctorant en histoire à l'Université de Ngaoundéré au Cameroun. Sa thèse en cours porte sur « L'artisanat du cuir dans l'Extrême-Nord du Cameroun du XIXe siècle à 2007 ॥.
} 


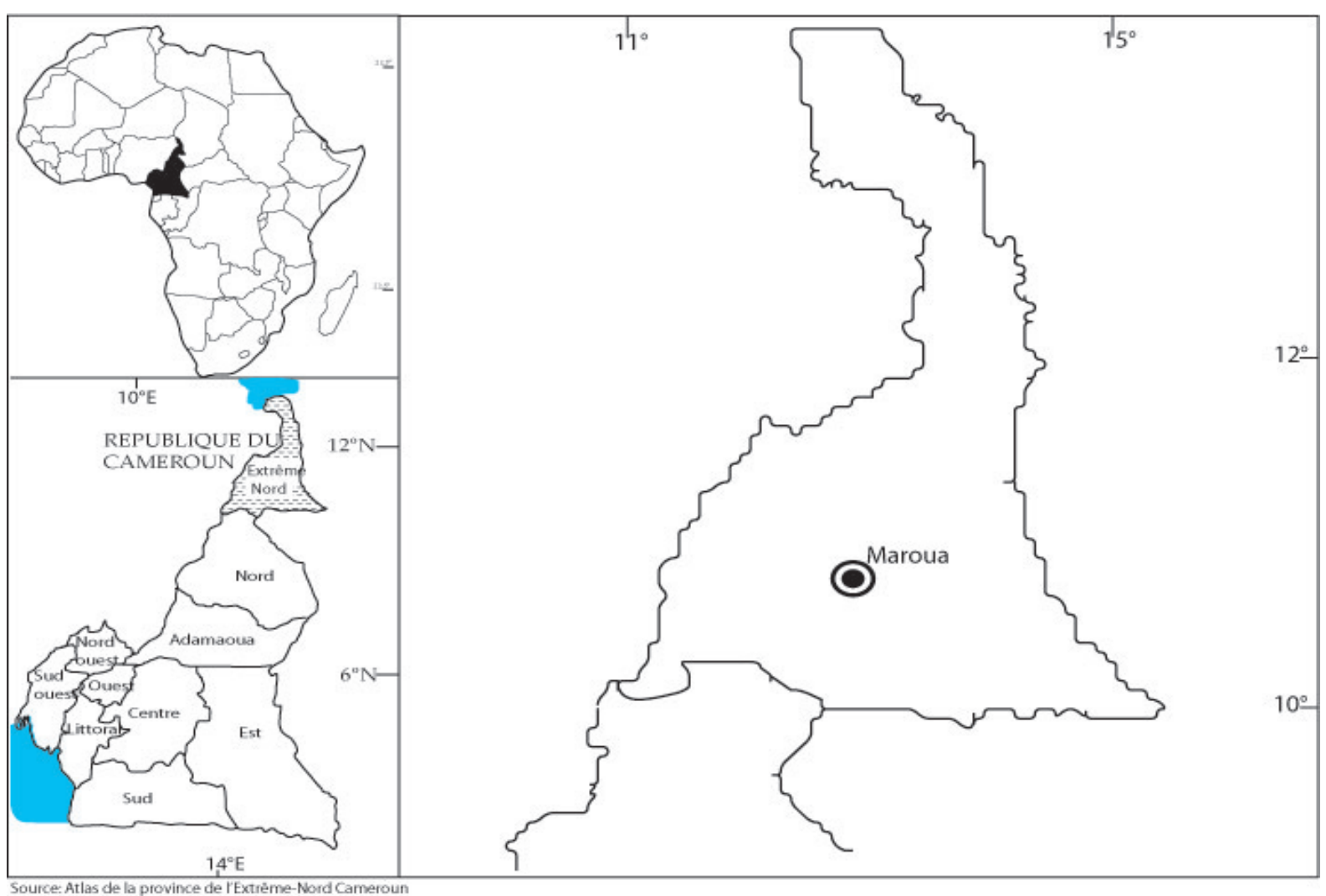

Carte de localisation de la ville de Maroua

Auteur : Félix Watang Ziéba

Eldridge Mohammadou ${ }^{3}$, Robert Nkili', Nicole Mainet et Paba Salé Mahamat5, Olivier Iyébi-Mandjek et Ghislaine Dégatierb, Christian Seignobos et Olivier lyébi-Mandjek ${ }^{7}$, Aïssatou-Boussoura-Garga ${ }^{8}$. Ce sont des ouvrages qui permettent d'avoir une vue

3 Eldridge Mohammadou, L'Histoire des Peuls Férôbé du Diamaré : Maroua et Pétté, Tokyo, ILCAA, 1976; id., "Islam et urbanisation dans le Soudan Central au XIXe siècle. La cité de Maroua (Nord-Cameroun)», The Proceedings of International Conference on Urbanism in Islam (ICUIT), Tokyo, 1989. 4 Robert Nkili, « Maroua, la ville et sa région, des origines à 1919 », thèse de doctorat de $3^{e}$ cycle en histoire, Université de Paris IV, 1976. 5 Nicole Mainet et Paba Salé Mahamat, " L'artisanat d'art à Maroua (Nord-Cameroun) "I, Annales de la Faculté des Lettres et Sciences Humaines $\_n^{\circ}$ 8, Yaoundé, 1977, pp. 159-186.

6 Ghislaine Dégatier et Olivier lyébi-Mandjek, «L'évolution de I'artisanat du cuir à Maroua ", rapport multigraphié, AFVP, 1992 ; Olivier lyébi-Mandjek, « L'artisanat du cuir à Maroua : étude de marché 11 , rapport multigraphié, 1993.

7 Christian Seignobos et Olivier lyébi-Mandjeck (éd.), Atlas de la province de L'Extrême-Nord, Paris, IRD, 2000 ; Christian. Seignobos, « De l'objet culturel au produit d'artisanat, de l'influence du tourisme au Nord-Cameroun 1), Enjeux, publication de la Fondation Paul Ango Ela, Yaoundé, n 5, 2006, pp. 30-35.

8 Aïssatou-Boussoura Garga, "Les mutations commerciales dans la région de Maroua pendant la première moitié du XXe siècle ", mémoire de maîtrise d'histoire, Université de Yaoundé l, 2000. globale sur le processus de développement de l'artisanat à Maroua, sur les filières existantes et de préciser les informations sur la période coloniale et sur celle de développement du tourisme à la fin des années 1960. Les données contenues dans ces études ne permettent cependant pas de comprendre suffisamment la touche des autorités coloniales françaises dans l'artisanat de Maroua, d'où la nécessité d'étudier les initiatives françaises en faveur de l'artisanat d'une part, et d'autre part de mesurer leur impact sur la dynamique de ce secteur. Autrement dit, il est question de s'interroger sur l'apport des autorités coloniales françaises dans le secteur de l'artisanat à Maroua et son impact sur l'évolution de cette activité locale.

Cette recherche s'appuie d'un côté sur l'exploitation des ouvrages, des mémoires, des thèses, des articles, des archives et de l'autre, sur des données orales collectées auprès des personnes qui ont une large connaissance de l'histoire de l'artisanat de Maroua. Ces données écrites et orales ont été complétées par des observations qui ont permis de se rendre compte d'un certain nombre d'investissements coloniaux dans le domaine de l'artisanat. La compilation, la confrontation des différents matériaux collectés et leur analyse critique a permis de tirer l'essentiel pour la construction de 
ce corpus articulé autour de trois points : l'artisanat à Maroua avant la colonisation ; l'organisation de l'artisanat par les autorités coloniales françaises; la contribution des autorités coloniales au commerce d'objets artisanaux à Maroua.

L'artisanat à Maroua avant la période coloniale française La présence française à Maroua comme dans tout le Cameroun remonte à l'année 1916 et dure jusqu'en 1960, date de l'indépendance?. C'est une période particulièrement importante pour l'artisanat de Maroua. En effet, ce secteur d'activité bénéficia de nombreuses innovations de la part des autorités françaises. Cependant, l'on ne saurait comprendre véritablement l'histoire de l'artisanat de Maroua sans se référer à l'histoire de la ville elle-même. Pour mieux saisir l'origine et le développement de l'activité artisanale à Maroua, il est important de remonter au XIXe siècle. Dans cette optique, il convient de s'intéresser d'abord à l'hégémonie peule dans la région au XIXe siècle avant de jeter un regard sur les filières mises en place et leur production.

L'hégémonie peule et la mise en place d'une industrie artisanale à Maroua au XIXe siècle

L'avènement de cette hégémonie ne saurait se comprendre sans qu' on ne fasse mention de quelques séquences de l'histoire même du Nord-Cameroun et des Peuls. Originaires de l'ancien empire du Mali (XIII _ $X V$ e siècles) connu sous le nom de Mallé, les pasteurs peuls entamèrent dès le XIVe siècle des migrations qui les amènent au Borno où ils séjournèrent pendant une longue durée auprès des Kanouri. Entre les XVIIe et XVIII siècles, de nombreuses factions peules entamèrent à nouveau des déplacements en direction cette fois du Fombina où ils s'implantèrent avec leurs troupeaux auprès des populations trouvées sur place à qui ils payaient tribut. Les relations entre ces groupes étaient pacifiques. II faut attendre le lancement du Jihad à partir de Sokoto par Ousman Dan Fodio en 1804 pour voir ces nouveaux venus prendre les armes contre les peuples trouvés sur place sous le prétexte de la propagation de l'Islam. C'est dans ce contexte que ces populations conquirent de vastes territoires dans ce qui deviendra le Nord-Cameroun où ils devinrent les principaux maîtres des lieux au détriment de ceux à qui ils étaient en quelque sorte soumis par le passé. Outre l'imposition de l'Islam et de l'hégémonie peule, il s'en est suivi une nouvelle organisation de la société avec la naissance de nouvelles entités dénommées Lamidats à la tête desquelles trônaient des Lamido,

9 Engelbert Mveng, Histoire du Cameroun, Paris, Présence Africaine, 1963. autorités très respectées. Au rang de ces entités créées et qui existent jusqu'à présent, figurent les Lamidats de Garoua, Ngaoundéré, Maroua, RayBouba, Bogo, Mindif, pour ne citer qu'elles ${ }^{10}$. Etant donné que l'étude porte sur Maroua, il s'avère indispensable de s'attarder sur l'évolution historique de cette cité qui permettra de mieux comprendre le développement de l'artisanat.

Avant l'avènement des Peuls, la zone appelée aujourd'hui Maroua fut successivement occupée, si l'on s'en tient aux découpages de l'histoire de cette cité faits par d'Eldridge Mohammadou, d'abord par les Mofu et puis les Guiziga entre les XVIIe et XVIII siècles. La période du XVIII siècle fut marquée par l'émergence de Marva"l avec les Guiziga. Les pasteurs peuls à la recherche des pâturages pour leurs troupeaux commencèrent à s'infiltrer peu à peu dans la région. La cohabitation fut pacifique au départ. Leur nombre s'accrut et c'est ainsi que les relations autrefois bonnes se détériorèrent. Entre 1792 et 1794 éclata un conflit entre les deux peuples, lequel sonna le glas du royaume Guiziga. Les Guiziga furent boutés hors de leur territoire tandis que se confirma l'hégémonie peule. Maroua devint alors une cité islamique avec la mise en place d'une dynastie peule. Sous le règne de Hamman Damraka (1801-1846), la ville connut une prospérité du fait de l'action de ce souverain. Dès lors prit naissance un commerce lucratif entre Maroua et le Bornou'2.

Parlant de ce commerce, Fisher fait part des caravanes chargées de marchandises diverses qui sillonnaient les deux royaumes. Tissus, natron, parfums, verroteries, papier étaient importés du Bornou tandis que Maroua exportait les peaux, l'ivoire, les esclaves et surtout les chevaux vers le Bornou. Tel qu'on le constate, l'importation et l'exportation se sont développées dans la cité peule en ce XIXe siècle. Ce dynamisme commercial ne fut pas sans conséquences. C'est ainsi que la population de Maroua s'accrut et cette zone devint

10 Au sujet de l'histoire des Peuls et du Jihad, les nombreux écrits de Eldridge Mohammadou sur ce peuple d'une part et les Lamidats du Nord-Cameroun d'autre part abondent en données. Outre ses travaux cités plus haut, il convient de citer aussi Les Lamidats du Diamaré et du Mayo-Louti au XIXe siècle, Tokyo, ILCAA, 1988 et " L'empreinte du Borno sur les Foulbé de l'Adamawa et leur langue ", Ngaoundéré-Anthropos. Revue de sciences sociales, vol. I, Yaoundé, Imprimerie Saint-Paul, 1996, pp. 71-90.

11 Terme guiziga d'où la dénomination Maroua tire son origine. 12 Cette synthèse de l'histoire de Maroua est extraite de E. Mohammadou, "Islam et urbanisation ", op. cit. note 3, pp. 117-154. 


\section{Autorités coloniales et économie artisanale à Maroua}

le poumon économique et la destination privilégiée des commerçants en provenance du Bornou et de l'ensemble du Caliphat de Sokoto's.

Une attention particulière mérite d'être portée sur les acteurs de ce négoce. C'étaient les Kanouri et les Haoussa qui firent de Maroua en ce siècle leur destination privilégiée. Plusieurs d'entre eux s'installèrent dans la cité peule où ils créèrent des entrepôts. II s'en suivit la naissance de plusieurs marchés. Sous leur impulsion naquit une véritable industrie artisanale du tissage, de la peausserie et de la forge. C'est dire que l'artisanat qui se développe en ce XIXe siècle a pour cheville ouvrière les Kanouri et les Haoussa ${ }^{14}$. Cela se comprend aisément Iorsqu'on s'intéresse à l'histoire de ces deux peuples, car l'on se rend compte qu'ils ont toujours fait dans la production artisanale. S'agissant des Kanouri par exemple, il est rapporté qu'ils comptaient parmi les artisans les plus expérimentés du Soudan occidental. Le Bornou d'où vient ce peuple connaît une intense activité artisanale depuis le XIVe siècle et même avant. Dans son ouvrage, Jacques Giri fait part de cette même activité dans les cités haoussa telles Kano, Katsina, Gobir ${ }^{15}$. Ces savoir-faire et bien d'autres ont été transférés dans le Nord-Cameroun en général et à Maroua en particulier grâce à la présence de ces deux peuples. A ce sujet, la conclusion d'Eldridge Mohammadou relative au rôle des Kanouri à Maroua est édifiante :

"Quoiqu'il en soit, le commerce et l'industrie à Maroua a été au XIXe siècle le fait d'abord des Kanouri [sic]. En bref, la cité de Maroua est une création bornouane autant que peule. Les Foulbé en ont jeté les bases, lui ont assuré son cadre politique, administratif, militaire et culturel, les Kanouri l'ont bâtie, nourrie, développée et enrichie, en organisant son industrie, son commerce et ses transports $11^{16}$.

Au total, la cité de Maroua connut un dynamisme remarquable au XIXe siècle au moment où s'installa l'hégémonie peule. Des populations diverses migrèrent vers cet eldorado commercial parmi lesquelles les Kanouri et les Haoussa qui mirent en

13 Fisher cité par E. Mohammadou, «I Islam et urbanisation », op. cit. note 3, p. 134. Le terme Caliphat renvoie à une entité ayant à sa tête un Calife qui est le descendant du Prophète Mahomet. 14 E. Mohammadou, «l Islam et urbanisation », op. cit. note 3, p. 134. 15 Jacques Giri, Histoire économique du Sahel, Paris, Karthala, 1994, p. 63.

16 E. Mohammadou , «Islam et urbanisation », op. cit. note 3, pp. 148-149. place un artisanat dont il importe d'examiner les filières développées et leur production.

Aperçu des filières mises en place et leur production Parlant de ces filières, Eldridge Mohammadou cite le tissage, le travail de la peau et la forge. Les artisans et commerçants kanouri et haoussa trouvèrent un terrain propice à l'exercice de leur art. Les débouchés étaient non négligeables du fait de l'accroissement de la population qui faisait de Maroua un vaste marché de consommation ${ }^{17}$.

Dans le domaine de la peausserie, tanneurs et cordonniers se mirent à l'œuvre. Les cuirs produits sur place servaient à confectionner des sacs pour le transport de mil, des selles et harnachements des chevaux, des gaines de couteaux, des couvertures de coran, des sandalettes pour bergers, des bottes pour cavaliers pour ne citer que ceux-là ${ }^{18}$.

La forge produisait des instruments aratoires tels que les houes, les arsenaux de guerre (les sabres, les couteaux, les lances, les pointes de flèche, etc.) ; la parure avec les objets comme les perles, les bijoux, les anneaux en fer ou en cuivre ${ }^{19}$.

Pour ce qui est du tissage, les tissus Leppi étaient produits à base du coton local. Ils permettaient de confectionner des tuniques de différentes sortes. II en était de même de longues bandelettes appelées Gabak et des Godon. Ces tissus servaient à confectionner surtout les boubous d'apparat destinés à la haute noblesse et à la bourgeoisie ${ }^{20}$. Aïssatou-Boussoura Garga classe d'ailleurs le coton et le fer du fait de leur importance dans la catégorie des monnaies " utilitaires" de l'époque ${ }^{21}$. E† à côté du tissage se développa la teinturerie, un autre type d'artisanat qui permet de colorer les tissus et habits cités plus haut 22 .

De ce qui précède, l'on se rend compte que les trois filières artisanales mises en place à Maroua produisaient de nombreux objets utilisés dans la région. Une lecture de cette production pousse à dire que cet artisanat revêtait les caractères

17 E. Mohammadou, "Islam et urbanisation ", op. cit. note 3, p. 134. L'auteur fait part de la prospérité de Maroua en ce moment avec la naissance de nouveaux quartiers et de plusieurs marchés.

18 G. Dégatier et O. Iyébi-Mandjek, op.cit. note 6, p. 2 ; A.-B. Garga, op.cit. note 8, p. 22.

19 Robert Nkili cité par Aïssatou-Boussoura Garga, op.cit. note 8, p. 22.

20 A.-B. Garga, op.cit. note 8, p.18 et p. 22.

21 Ibid, p. 17.

22 A.-B. Garga, op.cit. note 8, p. 23. 
politiques, militaires et religieux ${ }^{23}$. Les Peuls qui étaient détenteurs du pouvoir politique ne pouvaient trouver en cette activité qu'une initiative heureuse et admirable. En effet, pendant cette période où la culture occidentale n'avait pas encore commencé à influencer les populations africaines, l'artisanat était au centre de la vie desdites populations. Les produits des artisans étaient sollicités dans des domaines divers qui vont de l'agriculture, du matériel culinaire au vestimentaire en passant par les arsenaux de guerre. L'on comprend aisément pourquoil' aristocratie peule accordait un intérêt particulier aux métiers artisanaux d'où provenaient les tenues d'apparat portés par la classe aisée, les arsenaux de guerre utiles pour les armées des Lamidats et les l'équipement des chevaux qui étaient d'un grand intérêt pour les cours lamidales ${ }^{24}$.

Le développement de l'artisanat fut à l'origine de la naissance de nouveaux quartiers. Koutbao, Missingléo, Pidéré, Patchiguinari se peuplent alors de Bornouans se livrant au travail du cuir et des textiles ${ }^{25}$. L'hégémonie peule à Maroua et dans l'ensemble du Nord-Cameroun fut perturbée ou au mieux affaiblie par l'avènement de la colonisation, d'abord allemande de 1902 à 1914, date du début de la 1ère Guerre mondiale au cours de laquelle les Allemands furent chassés du Cameroun par les troupes alliées. Mais l'artisanat de Maroua n'aura pas été influencé par les Allemands. Deux tentatives d'explication peuvent être données à ce sujet.

Premièrement, Maroua opposa une farouche résistance à la conquête de la ville par les Allemands. Une bataille sanglante mit aux prises les armées du Lamido de Maroua et celles des Allemands à l'entrée de cette cité le 20 janvier 1902. C'est à l'issue de cette dernière que cette localité tombe sous la domination des Allemands qui instaurèrent dès lors une terrible répression à cause de la résistance des populations. Ce contexte d'insécurité et de terreur marquées par des tueries multiples perturba à plus d'un titre cette zone et entraîna d'ailleurs des migrations des populations vers d'autres horizons ${ }^{26}$. Etant donné que

\section{Ibid.}

24 Yougouda, doyen d'âge et Lawan de la tannerie traditionnelle de Madjema-Maroua, Kotoko et Ndjidda Bongo, Lawan en second de cette même unité de tannage, Showa, entretien du 28 février 2004 à Maroua.

25 C. Seignobos et O. Iyébi-Mandjek éd., op.cit note 2, p. 151. 26 A propos de la conquête de Maroua et d'autres aspects relatifs à la colonisation allemande à Maroua, les écrits des Allemands suivants constituent des sources importantes : Hans Dominik, 1902, "Expédition et combat contre Maroua ", TA-49, document consultable aux Archives Nationa- sans la tranquillité aucune activité n'est possible, une telle période n'était pas propice au déploiement de l'artisanat à une certaine échelle.

Deuxièmement, l'épisode allemand fut très bref (1902-1914). Ainsi, bien des chantiers auxquels tenaient ces premiers colonisateurs n'ont pu être réalisés. Mais à en croire Christian Seignobos et Olivier lyébi-Mandjek, l'idée d'organiser des corps de métiers dirigés par des chefs nommés par le Lamido tirerait son origine de la période allemande. II s'agissait d'une stratégie supplémentaire de contrôle de la population hostile à ces étrangers, en plaçant à la tête des différentes corporations des agents de renseignement acquis à la cause coloniale ${ }^{27}$.

Ce sont ces branches artisanales que les autorités coloniales françaises trouvèrent en place. Intéressées par ces savoir-faire, elles ne tardèrent pas à s'y impliquer.

\section{Les autorités coloniales, l'organisation et l'appui technique à l'artisanat}

Conscients de l'importance des métiers artisanaux de Maroua, les Français voulurent tôt les développer. Ils élaborèrent une politique dénommée « politique d'appui à l'artisanat ॥ qui consista dans un premier temps à organiser les différentes filières tout en y apportant des innovations techniques ${ }^{28}$.

En ce qui concerne l'organisation, les administrateurs conseillèrent au Lamido de chercher des artisans aptes et de les nommer à la tête des différentes corporations. C'est ainsi qu'à la tête de celles-ci furent nommées des personnes auxquelles les titres de Lawans ${ }^{29}$ qui est encore d'actualité furent attribués comme s'il s'agissait des responsables d'entités politiques quelconques. La maroquinerie, la tannerie, la broderie, le tissage, la cordonnerie par exemple furent ainsi organisés. La transposition de ce titre au niveau de l'artisanat montre l'importance de

les de Yaoundé ; Zimmerman, Durch Busch und Steppe, von Campo bis zum Schari 1892-1902; E. Mohammadou, «Islam et urbanisation 11, op. cit. note 3, pp. 139-142.

27 C. Seignobos et O. lyébi-Mandjek éd., op.cit. note 2, p. 160. 28 O. Iyébi-Mandjek, op.cit. note 6, p. 4.

29 Dans la première articulation de cette réflexion, le terme Lamido a été expliqué, de même que le contexte de son avènement. II a été dit qu'un Lamido trône à la tête d'un Lamidat, entité qui est constituée de plusieurs Lawanats. Ces dernières entités qui sont en dessous des Lamidats sont généralement formées d'un conglomérat de villages placés sous l'autorité d'un Lawan. Dans le contexte précis, il ne s'agit pas des Lawans qui commandent des entités quelconques, mais des personnes à qui on a donné la responsabilité des filières artisanales. 


\section{Autorités coloniales et économie artisanale à Maroua}

ce secteur pendant cette période. S'il faut donner une explication au choix porté sur le Lamido par les Français, il faut savoir que l'environnement ainsi étudié est très spécifique. Cette autorité traditionnelle a une forte influence sur ses sujets qui lui doivent un respect inconditionné. II était donc la personne la mieux indiquée pour faire réussir l'application de la politique ainsi élaborée dans le domaine de l'artisanat.

La fonction de ces responsables des filières artisanales consistait à répartir les commandes d'objets artisanaux en provenance des autorités françaises qui passaient par la chefferie traditionnelle dirigée par le Lamido. Celle-ci chargeait à son tour les Lawans de faire exécuter les tâches ${ }^{30}$. Ces derniers étaient à proprement parler les patrons des filières et représentaient directement le pouvoir traditionnel, c'est-à-dire le Lamido qui avait une influence sur toutes les activités de sa zone de commandement. Tel était le système de commercialisation où tout était régi par la chefferie. L'artisanat était donc un secteur hautement politisé. Comme l'argent des commandes passait par plusieurs canaux, certaines sources disent qu'il arrivait parfois que l'argent des artisans était soit détourné par le Lamido avec la complicité des Lawans $^{31}$. Ce qui frustrait ces maîtres de l'art qui ne pouvaient se plaindre du fait de la considération et du respect qu'ils devaient au Lamido.

II ressort de ce qui précède que, dans une perspective de promotion de l'artisanat local, les autorités françaises s'appuyèrent sur l'autorité traditionnelle incarnée par le Lamido à qui elles demandèrent d'organiser les différentes filières avec la nomination à leurs têtes des chefs appelés Lawans. Ainsi, les directives de l'administration coloniale dans le domaine de l'artisanat passaient par le Lamido et ses collaborateurs qui servaient ainsi de courroies de transmission.

Sur le plan technique, les Français estimaient que l'artisanat de Maroua avait des insuffisances qui empêchaient son exploitation à des fins économiques. En effet, les produits artisanaux n'intéressaient pas la clientèle aisée constituée surtout d'Européens de la place. La production était plutôt tournée vers la population locale avec la confection d'objets susévoqués, en relation avec le milieu. Cela ne faisait pas la fierté des colons. II fallait, selon eux, intégrer cette activité de Maroua dans l'économie moderne. Pour ce faire, il fallait " civiliser " l'artisanat en initiant ses acteurs à des nouvelles techniques. C'est ainsi

30 O. Iyébi-Mandjek, op.cit. note 6, p. 5 ; C. Seignobos et O. lyébi-Mandjek éd., op.cit. note 2, p. 160 font part de cette organisation des corps des métiers à Maroua dont l'artisanat. 31 O. Iyébi-Mandjek, op.cit. note 6, p. 5. que des artisans de Maroua séjournèrent en France et au Maroc. Cependant, il semble que leur séjour fut un échec dans la mesure où les artisans formés ne purent pas vulgariser les techniques acquises à leur retour du fait de la réticence de leurs pairs restés sur place à Maroua ${ }^{32}$.

Les colons poussèrent les artisans à la fabrication de nouveaux modèles d'objets inspirés de l'art marocain et européen. Dans le secteur du cuir par exemple, les poufs, les babouches et les sandalettes à boucles, les mallettes, les porte-documents, pour ne citer que ceux-là remplacèrent les produits antérieurement fabriqués. C'est à cette époque que les artisans du cuir par exemple commencèrent à utiliser les peaux d'animaux sauvages et reptiles pour confectionner des objets divers ${ }^{33}$. Dans le domaine des textiles, les nappes de table, les habits inspirés des modèles européens furentà la mode. L'administration coloniale confia par exemple la confection des uniformes des prisonniers, des tenues pour personnel de l'hôpital ainsi que des serviettes de cet établissement aux artisans. L'objectif des Français était clair : il fallait à tout prix détourner l'artisanat de son orientation locale vers une clientèle européenne. C'est à juste titre que Christian Seignobos et Olivier Iyébi-Mandjeck Olivier relèvent qu' " à l'époque coloniale, cordonnerie et maroquinerie ont été orientées vers la satisfaction d'une clientèle européenne ${ }^{34}$.

L'action européenne se situa aussi au niveau des privilèges accordés aux artisans. C'est ainsi par exemple que les cordonniers et maroquiniers furent exemptés des corvées de voirie autrefois obligatoires. Cela montre à quel point les autorités françaises avaient accordé de l'importance à l'artisanat en cette période. Pareil traitement ne pouvait que résoudre les acteurs de ce secteur d'activité à s'y intéresser davantage. Toutes ces actions en faveur de l'artisanat de Maroua eurent des retombées ${ }^{35}$.

II s'ensuivit en effet une augmentation du volume de la production artisanale à Maroua. Les produits artisanaux connurent dès lors un succès auprès des Européens résidant dans la Région du Nord-

32 O. Iyébi-Mandjek, op.cit. note 6, p. 4. Relativement à l'envoi des artisans de Maroua à l'étranger la plupart des informateurs rencontrés infirment plutôt ce point de vue. La consultation d'archives et bien d'autres documents dans nos recherches ultérieures permettront peut-être de lever cette équivoque.

33 François Wassouni, « Production, consommation et commercialisation du cuir à Maroua : XIX $X^{e}-X X^{e}$ siècles 1 , mémoire de maîtrise d'histoire, Université de Ngaoundéré, 2002, p. 79. 34 C. Seignobos et O. Iyébi-Mandjek éd., op.cit. note 3, p. 33. 35 Ibid. 
Cameroun et de l'ensemble du territoire camerounais qui constituaient ainsi l'essentiel de la clientèle de ces nouveaux produits. Progressivement, ces objets fabriqués conquirent tout de même des marchés hors des frontières du Cameroun, notamment dans les autres territoires français d'Afrique (Afrique de l'Ouest et Afrique Équatoriale Française) et en Europe. Des commandes des produits artisanaux en provenance de tous ces horizons cités étaient adressées au Lamido qui les faisait exécuter par les artisans. Une fois les produits confectionnés, ils étaient livrés aux autorités françaises qui faisaient des colis, lesquels étaient expédiés par le biais de véhicules et d'avions en direction des destinations d'où venaient ces commandes ${ }^{36}$. Parallèlement à cette forme de distribution, la demande grandissante d'objets artisanaux a résolu quelques commerçants européens exerçant dans la partie nord du Cameroun à s'investir dans ce secteur de commerce. Ils achetaient les produits, puis les acheminaient vers d'autres localités du territoire camerounais, vers d'autres destinations extérieures à l'instar du Tchad et de l'Europe. A titre d'exemple, Olivier lyébi-Mandjeck cite un certain Mathey qui montait des caravanes pour acheminer les produits en cuir collectés à Maroua vers le Tchad $^{37}$. Tout cela démontre l'importance qu'a pris l'artisanat en ce moment. D'un artisanat tourné vers une clientèle locale, l'on passa à un artisanat orienté vers la satisfaction des intérêts européens. Ce qui n'est pas sans conséquences sur la population locale qui s' approvisionnait en objets usuels nombreux auprès des artisans qui, du fait des dividendes que rapportait désormais leur activité et de nombreux autres privilèges accordés par les Français, fabriquaient davantage pour la clientèle européenne. Les objets fabriqués perdirent dès lors leur caractère traditionnel, d'où l'acculturation de l'artisanat et le recul du savoir-faire purement local. Cela ressort d' ailleurs sous forme de déploration dans un rapport qui mentionne : «Si les produits flattent le goût européen, ils n'ont plus qu'un lointain rapport avec l'art

36 Boukar Godjé, ancien agent d'entretien du Centre Artisanal de Marova actuellement en service à la Société Coopérative d'Épargne et de Développement (SOCOOPED), Mandara, entretien du 28 janvier 2004 à Maroua. A cette époque, il existait des aérodromes dans la périphérie de Maroua, notamment à Salak et à Kaélé qui expédiaient la viande en direction de la partie méridionale du Cameroun, des autres territoires et d'Europe. Voir Henri Fréchou, L'élevage et le commerce du bétail dans le Nord-Cameroun, Cahiers de L'ORSTOM, Série sciences humaines ${ }_{\iota}$ Vol. III, $n^{\circ} 2$, 1966, pp. 93-94.

37 O. Iyébi-Mandjek, op.cit. note 6, p. 5. indigène. Ce qui est regrettable $\|{ }^{38}$.

Comment comprendre cet activisme des Français dans la promotion de l'artisanat ? S'agissaitil réellement d'une volonté de développer cet art qui avait pendant des siècles été frappé d'une connotation négative et caractérisé d'art "primitif », " sauvage " ? ${ }^{39}$ En réalité, tout semble montrer que l'objectif de cette entreprise française était de tirer les dividendes des objets artisanaux en faisant d'eux des marchandises qui pouvaient intéresser les Européens vivant au Cameroun, dans les autres territoires et en Europe. Dans les rapports administratifs, on se plaignait de l'absence d'une activité commerciale digne dans la Subdivision. II fallait donc tout au moins stimuler les secteurs à même de rapporter quelque chose parmi lesquels l'artisanat dont les produits intéressaient de nombreux Européens. Le chef de cette Subdivision, Soupault, soulignait en 1945 ce qui suit à propos du commerce : " mis à part le commerce du bétail et des peaux d'une part et celui des objets ouvrés en cuir d'autre part, le trafic commercial de la Subdivision est pratiquement nul ». Aussi convient-il de préciser que l'on se situe dans un contexte où la culture des peuples colonisés avait acquis une certaine reconnaissance par les colonisateurs et un certain nombre de mesures de protection étaient en train d'être prises. De plus, un intérêt pour les productions d'objets des peuples soumis se manifestait en Europe. Lesdits objets étaient sollicités par des particuliers d'une part et les Musées d'autre part qui en faisaient des collections ${ }^{40}$. Le marché d'objets artisanaux était prometteur et donc susceptible de faire rentrer des devises aux structures qui s'y investissaient.

Les autorités coloniales se sont donc investies dans le développement de l'artisanat de Maroua. Les actions en sa faveur allèrent de l'organisation des filières à l'introduction de nouveaux modèles

38 Rapports annuels, Maroua, 1940-1951 cités par G. Dégatier et O. Iyébi-Mandjek, op.cit. note 6, p. 17.

39 Au sujet des préjugés sur l'art africain, l'on voudra bien consulter les ouvrages de Frank Willett, L'art africain, traduction française, Paris, Thames \& Hudson, 1990 ; Jacques Kernache, Jean-Louis Paudrat et al., L'art africain, Paris, Mazenod, 1988 ; Joseph Adandé, " L'art africain et l'imaginaire des autres entre le $X V l^{e}$ et le début du XXe siècle. Essai d'analyse diachronique des prémisses d'un processus de " globalisation », Afrika Zamani, nos 9 et 10, 2002, pp. 60-76.

40 Etienne Féau, «L'art africain dans les collections publiques européennes ", dans Caroline Gaulthier-Kurhan éd., Le patrimoine culturel africainŁ Paris, Maisonneuve et Larose, 2001, pp. 229-295 ; Vincent Négri, « La création du droit du patrimoine culturel en Afrique "), dans Caroline Gaulthier-Kurhan, éd., Le patrimoine culturel africain, Paris, Maisonneuve et Larose, 2001, pp. 321-340. 


\section{Autorités coloniales et économie artisanale à Maroua}

d'objets, en passant par l'accord d'importants privilèges sociaux aux artisans. C'était sans doute une façon de les encourager. La production artisanale se densifia, mais les objets fabriqués pour la plupart n'avaient plus rien à voir avec le passé. Ils étaient destinés non plus aux populations locales, mais à une clientèle européenne vivant dans le périmètre de Maroua, dans le territoire camerounais, dans d'autres colonies françaises d'Afrique et en Europe. Mais la commercialisation de la production artisanale nécessitait la mise sur pied d'infrastructures relatives. Les Français ne tardèrent pas à s'investir dans ce projet. Tout commença par la création d'une section de vente de ces produits par la SIP qui sera suppléée par d'autres structures plus importantes.

\section{Les autorités coloniales françaises et la mise en place des} infrastructures de commerce d'objets artisanaux à Maroua Entre 1947 et 1955, les Français s'investissent davantage dans le secteur de l'artisanat, non plus dans le domaine technique et organisationnel, mais dans la commercialisation des produits. C'est ainsi qu'ils vont créer des structures de vente d'objets artisanaux. La création de ces structures comprend deux phases. La première est marquée par l'ouverture d'une section artisanale par la Société indigène de prévoyance (SIP) tandis que la deuxième phase est celle de la création du Centre artisanal.

La Société indigène de prévoyance (SIP) et la section artisanale de Maroua

Le système de commercialisation centralisé par le Lamido a été déjà analysé. II en ressort que ce dernier influençait souvent négativement les filières artisanales en privant les artisans de leur argent, d'où leur découragement. Les Français voulant s'investir eux-mêmes dans le commerce d'objets artisanaux, ils supprimèrent cet intermédiaire. En 1947, la SIP, organisme coopératif qui faisait le commerce pendant la période coloniale française, ouvrit ainsi une section artisanale à Maroua à l'emplacement des bureaux de l'actuelle province alors siège de la Région ${ }^{41}$. Rappelons que les premières coopératives sur le territoire du Cameroun furent créées à partir de 1925 à l'initiative du Gouverneur Marchand. Leurs objectifs se trouvèrent améliorés par la création des SIP instituées par arrêté du 7 juin 1937, promulgué le 7 juillet de la même année ${ }^{42}$. Ainsi, avec l'avènement de cette structure commerciale, les artisans ne passaient plus par le Lamido, mais acheminaient

41 C. Seignobos et O. Iyébi-Mandjek.éd., op.cit. note 2, p. 162 ; O. Iyébi-Mandjek, op.cit. note 7, p. 6.

42 Guernier cité par A.-B. Garga, op.cit. note 9, p. 73. directement leurs produits au niveau de ladite section une fois qu'ils avaient fini de les fabriquer. Les livraisons se faisaient entre une et trois fois par semaine et mettaient les artisans en contact direct avec les responsables de cette agence de vente. Une fois les produits vendus à l'issue du marchandage, les artisans recevaient immédiatement leur argent ou un reçu leur était donné et ils pouvaient passer à la caisse après quelques jours. On ne peut conclure que les Lawans n'existaient plus dans les filières artisanales. Ils assistaient aux différentes ventes, mais ils n'avaient plus le monopole d'antan de même que le Lamido qui ne recevait plus que de temps à autre quelques sommes en guise de Kola de la part des artisans ${ }^{43}$. La mise à l'écart de ce dernier ne pouvait en aucun cas le rendre heureux dans la mesure où il trouvait par le passé son compte dans les transactions commerciales. Mais, il ne pouvait s'opposer à la décision de l'administration coloniale à qui il était soumis.

L'antenne de la SIP de Maroua faisait le commerce de plusieurs articles. Elle avait dans ce sens des sections commerciales parmi lesquelles celles de la vente d'essence, les magasins des grains auxquels s'ajouta la section de vente des produits artisanaux. La traite des arachides fut aussi l'une des activités de cette société. Elle visait aussi à travers ses activités des buts sociaux en créant des réserves alimentaires pour pallier les éventuelles disettes en venant en aide, en cas de besoin, aux indigènes. Dans cette perspective, elle stocka par exemple 164 tonnes de mil en $1949^{44}$. Cette structure avait donc une activité commerciale diversifiée.

La SIP intervenait dans le commerce des produits artisanaux, encourageait la production en achetant elle-même ces produits. Elle le faisait aussi bien dans le but de faire la promotion de l'artisanat de Maroua que dans un but purement lucratif ${ }^{45}$. Les rapports annuels de 1940-1951 évoquent ses activités en ces termes : « La SIP achète aux artisans du cuir et aux brodeurs pour vendre à la clientèle européenne ou africaine de la région et de tout le territoire (...) ou exporter en métropole $1{ }^{46}$.

A l'issue de cette présentation, l'on constate que les activités de la SIP étaient importantes. Aussi dépassaient-elles le cadre de Maroua pour s'étendre

43 Yougouda, doyen d'âge et Lawan de la tannerie traditionnelle de Madjema-Marova, Kotoko et Ndjidda Bongo, Lawan en second de cette même unité de tannage, Showa, entretien du 28 février 2004 à Maroua

44 A.-B. Garga, op.cit. note 9, pp. 73-75.

45 ANY, APA 11618, Rapports annuels 1949.

46 ANY, Rapports annuels 1940-1951. 
au-delà même du territoire du Cameroun. C'est dire que les débouchés étaient conséquents. On peut comprendre dès lors pourquoi les Européens s'étaient évertués à donner une orientation nouvelle à l'artisanat.

Dans le cadre de la promotion de l'artisanat, la SIP assurait aux artisans un débouché rémunérateur pour les meilleures productions. E† dans le même sens, un label de qualité était octroyé à tout article proposé à l'achat afin de ne pas frustrer les artisans. C'était sans doute une stratégie d'encouragement des artisans qu'on exhortait par la même occasion à perfectionner leur art.

Sur le plan de l'organisation, l'appareil directeur de la SIP avait pour président le chef de la Région. La section artisanale, annexe de la SIP, était gérée par une directrice européenne, généralement l'épouse même du chef de la Région, comme la femme de Bertrand Lembezat, qui a été la toute première. II apparaît dès lors que cette structure de commercialisation des produits artisanaux était importante du fait de l'implication des hautes personnalités de la place. Leur attention envers ce négoce s'expliquerait par l'existence des marchés extérieurs mentionnés plus haut, aux débouchés importants. Ce qui permettait d'engranger des bénéfices. Mettre au Centre de cette structure un proche de la haute autorité de la place n'était donc pas étonnant.

La section de vente d'objets artisanaux ainsi mise en place à Maroua en 1947 ne tarda pas à prospérer. La demande sans cesse croissante des produits artisanaux lui donna une renommée ${ }^{47}$. Le bilan des ventes de 1949 de cette section de la SIP fait ressortir son importance en ces termes : "Elle a ouvert un magasin de vente très prospère qui est maintenant connu dans tout le territoire. Le montant total des ventes s'est élevé au cours de l'année 1949 à 700021 francs $\gg{ }^{48}$.

L'artisanat devint un secteur d'activité important de la ville de Maroua. L'administration coloniale ne cessa de multiplier des projets dans ce domaine. Ce fut le cas de l'idée de la création d'une école artisanale à Maroua avec pour objectif de « former des artisans capables de faire revivre les arts indigènes régionaux $1{ }^{49}$. Dans la même perspective,

47 O. Iyébi-Mandjek, op.cit. note 7, p. 6. Cette renommée a été mentionnée plus haut avec la conquête d'importants marchés hors des frontières du Cameroun (Europe, Afrique de l'Ouest, AEF...)

48 ANY, APA 11618, rapport 1949

49 Cette idée est rapportée par G. Dégatier et O. Iyébi-Mandjek, op.cit. note 7, p. 16. Les deux auteurs citent les rapports il convient de noter le premier recensement des artisans de Maroua en 1948. Au total, 574 artisans furent recensés, soit 200 tisserands, 44 teinturiers, 100 tanneurs, 30 cordonniers, 100 forgerons et 100 potiers ${ }^{50}$. Un autre travail de ce genre fut réalisé par Prestat qui recensa 79 tanneurs, 74 cordonniers, 141 maroquiniers, soit un total de 290 artisans. C'était un recensement axé essentiellement sur les filières du cuir ${ }^{51}$. Cette initiative démontre une fois de plus l'intérêt des Français pour l'artisanat. L'on s'imagine qu'avec les chiffres avancés, la production serait vraiment importante même si tous les artisans ne produisaient pas uniquement pour la SIP. Une partie satisfaisait de temps à autre la population locale en fabriquant les objets utilitaires d'antan

En parlant de cet artisanat de Maroua, il a été davantage question des objets issus du travail du cuir et du secteur des textiles. Cela ne signifie pas que les autres filières de l'artisanat n'existaient pas. Seulement, les autorités françaises s'étaient davantage intéressées aux secteurs mentionnés ci-dessus. Ce sont d'ailleurs eux qui font jusqu'à aujourd'hui la renommée de l'artisanat de Maroua.

La SIP fut pendant longtemps, avec la R.W. King ${ }^{52}$, les seules structures de commerce à Maroua ${ }^{53}$. Elle fit la vente de ces objets artisanaux dans sa section jusqu'en 1955, date à laquelle le Centre artisanal lui emboîta le pas.

L'avènement d'un Centre artisanal à Maroua La création de cette structure se situe à une période où l'artisanat de Maroua avait acquis ses lettres de noblesse avec l'exportation de ses produits au-delà des frontières du Cameroun. C'était en quelque sorte l'aboutissement de la politique d'appui à l'artisanat conçue par les autorités coloniales françaises à partir des années 1940. Du fait de la croissance de la ville et de la production d'objets d'art, il fallait mettre en place un cadre dépassant la petite section créée par la SIP. Ladite structure devrait être capable de contenir la production artisanale de la région. Le projet de construction du Centre Artisanal ne tarda pas à se concrétiser.

annuels de la Subdivision de Maroua, 1940-1951.

50 Rapport annuel 1949, Subdivision de Maroua, ANY, APA 11747 cité par Marcel Roupsard, Nord-Cameroun. Ouverture et Développement, Coutances, Claude Bellée, 1987, p. 456. 51 Gérard Prestat, cité par G. Dégatier et $O$. Iyébi-Mandjek, op.cit. note 7, p. 3.

52 C'est une société anglaise très active dans le territoire camerounais pendant la période coloniale et qui disposait d'une agence à Maroua.

53 C. Seignobos et O. Iyébi-Mandjek,. éd., op.cit. note 3, p. 54. 


\section{Autorités coloniales et économie artisanale à Maroua}

Si l'on s'en tient aux informations orales, le Centre artisanal a été créé au cours du règne du Lamido Yaya Daïrou qui va de 1943 à $1958^{54}$. Les sources écrites portant sur la ville de Maroua donnent une précision quant à la date de création de cet édifice pendant la période française en avançant l'année 195555. II n'existe donc pas un grand décalage entre ces données orales et écrites. La mise en place du Centre bénéficia du concours de plusieurs acteurs. Ce furent les autorités coloniales françaises, les autorités traditionnelles de Maroua et ses environs, sans toutefois oublier les artisans.

L'administration coloniale française contribua largement à cette œuvre puisqu'elle en est l'initiatrice. Aussi ne ménagea-t-elle aucun effort pour sa réalisation. Nous évoquions tantôt la politique conçue par les autorités françaises pour appuyer l'artisanat. Cela eut pour résultat l'augmentation du volume de production d'objets d'art qui incita ces autorités à créer ledit Centre. L'administration française en fut le maître d'ouvrage. La SIP qui fut active dans la stimulation de la production artisanale et le commerce des produits dérivés était un organisme français. Devenue Société africaine de prévoyance (SAP) au moment de la création du Centre Artisanal, elle était sans doute en étroite relation avec l'administration coloniale puisque son Président était le Chef de Région ${ }^{56}$. Au total, les autorités françaises jouèrent un rôle capital dans le processus d'édification de cette structure.

L'apport des autorités traditionnelles ne fut pas

54 C'est ce qui ressort des entretiens avec Yougouda, doyen d'âge et Lawan de la tannerie traditionnelle de Madjema-Maroua, Kotoko et Ndjidda Bongo, Lawan en second de cette même unité de tannage, Showa, entretien du 28 février 2004 à Maroua ; Boukar Godjé, ancien agent d'entretien du Centre Artisanal de Maroua actuellement en service à la SOCOOPED, Mandara, entretien du 28 janvier 2004 à Maroua. Au sujet du règne de ce Lamido, consulter Abdouraman Halirou, "Yaya Daïrou, Lamido de Maroua (1943-1958) I1, mémoire de maîtrise d'histoire, Université de Ngaoundéré, 1997.

55 C'est ce qui ressort des travaux des auteurs tels que Paba Salé Mahamat, « Maroua : aspects de la croissance d'une ville du Nord-Cameroun » (Des années 50 à nos jours), thèse de doctorat de $3^{e}$ cycle de géographie, Université de Bordeaux III, 1980, p. 176 ; C. Seignobos et O. Iyébi-Mandjek éd., op.cit. note 3, p. 162 ; Mohammadou Bachirou, «Tannage et dynamique socio-économique à Maroua : 1801-1997 "), rapport de licence d'histoire, Université de Ngaoundéré, 1997, p. 22. 56 Marcel Roupsard, op.cit. note 51, p. 437. L'auteur mentionne d'ailleurs l'influence de ces administrateurs coloniaux dans les Sociétés de Prévoyance créées à Garoua, Ngaoundéré et Maroua. négligeable. Le Lamidat de Maroua servit de relais dans la politique d'encadrement de l'artisanat. ॥ joua le rôle de sensibilisation des artisans. Les Lawans placés par le Lamido répercutaient les directives dans les filières respectives. Le Lamido Yaya Daïrou joua un rôle de premier plan en aidant les Français à mettre en place ce Centre ${ }^{57}$. Aujourd'hui, la communauté des artisans le considère comme un pionnier essentiel de ce centre culturel et artistique. Son rôle et son implication sont ancrés dans la mémoire collective à Maroua et on raconte à ce sujet ce qui suit :

Le Lamido Yaya Daïrou aimait beaucoup la culture et surtout les arts. II fit appel aux artisans de son Lamidat dans le cadre d'une exposition d'objets d'art. II invita pour la circonstance les autorités françaises de Maroua qui en furent émerveillées. Fascinées par la magnificence de ce génie artisanal, elles décidèrent de faire construire une structure d'exposition et de commercialisation des objets d'art de Maroua. Ce qui permettrait à tous les étrangers de passage dans la région d'apprécier à sa juste valeur ce secteur d'activité florissant. Eu égard au caractère historique du site ayant servi autrefois de marché d'esclaves, le Lamido exigea que le Centre y soit construit. Dans ses intentions, ce monument devrait servir de lieu de mémoire pour les générations à venir ${ }^{58}$.

Si ces informations doivent être prises avec prudence, elles font tout de même part du rôle joué par Yaya Dairou dans cette entreprise. C'est d'ailleurs dans les années 1940, début du règne de ce souverain que l'artisanat de Maroua a acquis sa renommée comme nous l'avons vu plus haut59. Au-delà d'une simple maison d'exposition et de vente des objets artisanaux, d'un centre culturel, le Centre Artisanal est un lieu de mémoire autour duquel se cache un pan important de l'histoire de la région qu'est le commerce d'esclaves. Mais c'est une réalité

57 Boukar Godjé, ancien agent d'entretien du Centre Artisanal de Marova actuellement en service à la SOCOOPED, Mandara, entretien du 28 janvier 2004 à Maroua.

58 C'est ce qui ressort des entretiens que nous avons eus avec des artisans âgés de Maroua. II s'agit de Yougouda, doyen d'âge et Lawan de la tannerie traditionnelle de MadjemaMaroua, Kotoko et Ndjidda Bongo, Lawan en second de cette même unité de tannage, Showa, entretien du 28 février 2004 à Maroua ; Boukar Godjé, ancien agent d'entretien du Centre Artisanal de Maroua actuellement en service à la SOCOOPED, Mandara, entretien du 28 janvier 2004 à Maroua. 59 Abdouraman Halirou, op. cit. note 55, p. 36 mentionne l'artisanat au rang des activités importantes et prospères durant le règne de ce souverain. 


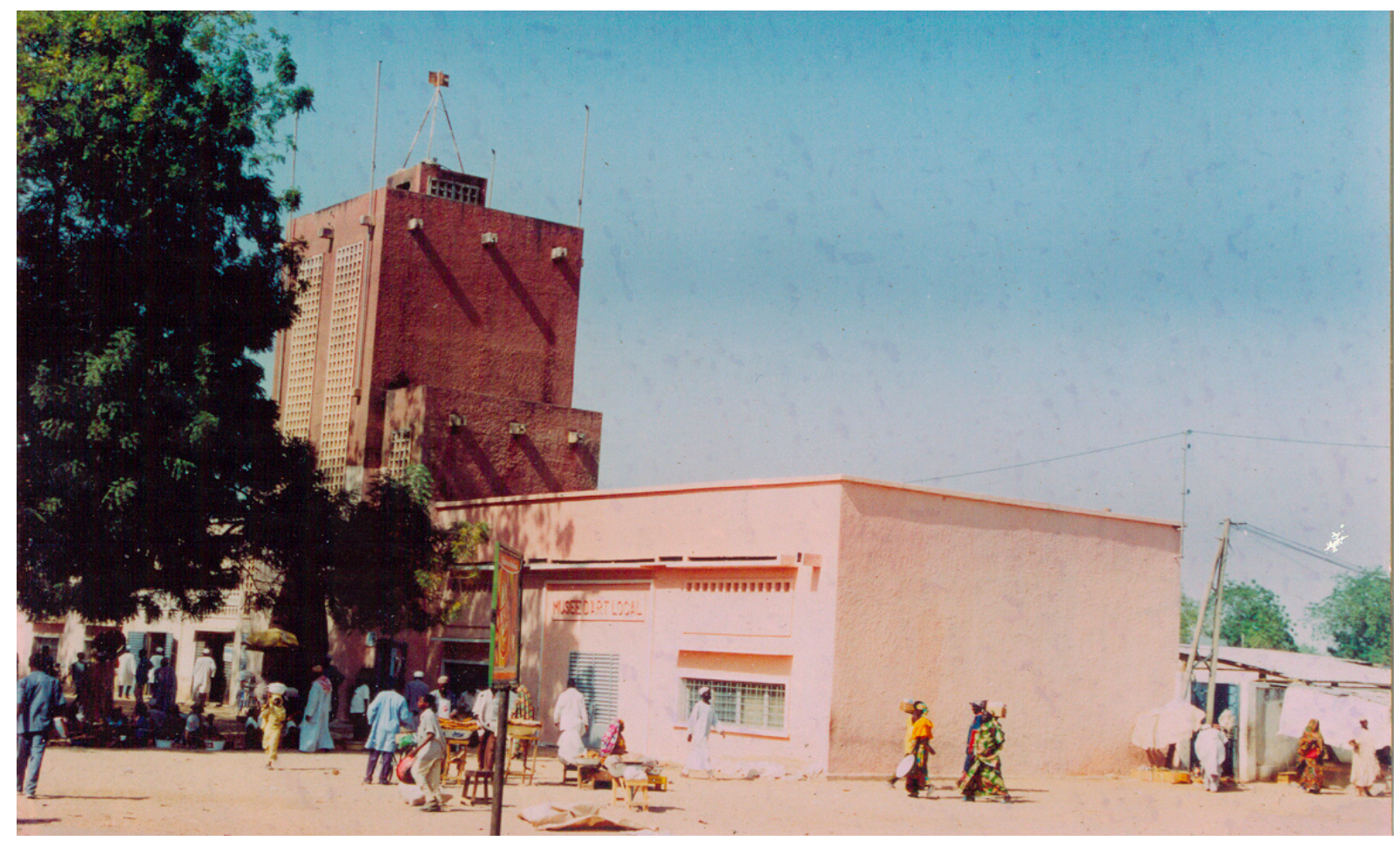

Figure 1- Une vue d'ensemble du Centre Artisanal de Maroua, une des réalisations d'envergure des autorités coloniales françaises dans le domaine de l'artisanat. On peut apercevoir la grande tour qui s'élève au-dessus de cet établissement. (c) F. Wassouni, janvier 2004.

qui semble être méconnue par beaucoup jusque-là 60 .

L'on ne saurait évoquer les acteurs sans mentionner les artisans de Maroua. Ce sont eux qui ont fasciné les Français par leur génie, mieux leur savoir-faire. Ce qui résolut ces derniers à s'intéresser à leur corporation ${ }^{61}$. Ils constituaient la cheville ouvrière de tout ce processus.

Les autres autorités traditionnelles des environs de Maroua ne furent pas en dehors de ce chantier. Il est rapporté qu'avant le lancement des activités du Centre artisanal, elles furent convoquées par les Français. C'était dans le but de leur expliquer le bien-

60 Sur la création de cet édifice colonial qui occupe une place de choix parmi les sites touristiques de cette ville, voir le travail de François Wassouni : " L'artisanat dans l'Extrême-Nord du Cameroun : XIX ${ }^{e}-X X^{e}$ siècles $॥$, mémoire de DEA d'histoire, Université de Ngaoundéré, 2004.

61 Boukar Godjé, ancien agent d'entretien du Centre Artisanal de Maroua actuellement en service à la SOCOOPED, Mandara et Hamadou Halilou, agent de la SOCOOPED et fils de l'interprète du Centre Artisanal de Maroua, Guiziga, entretien du 28 janvier 2004 à Maroua ; Mahamat Chérif, responsable du Centre Artisanal de Maroua, Kanouri, entretien du 25 janvier 2004 au Centre Artisanal. fondé de la structure qui allait être mise en place. Elles devaient par conséquent exhorter les artisans de leurs zones respectives à acheminer leurs produits artisanaux vers Maroua ${ }^{62}$. Ce qui signifie que ce Centre n'était pas conçu pour n'abriter que les seuls produits de l'artisanat de Maroua. Certains Lamibéb3 collectionnèrent les objets d'art de leurs zones et les offrirent aux autorités françaises. Ces objets, une fois rassemblés, furent déposés dans le Musée d'art local de Maroua. C'est à partir de la collection d'objets d'art des différentes localités du Diamaré que ce Musée d'art local de Maroua fut élaboré.

Le Centre artisanal fut ainsi construit à l'entrée de l'actuel grand marché de Maroua, en face du quartier Founangué. Ce fut au départ une bâtisse d'une quarantaine de mètres de long et d'une dizaine de large divisée en deux parties inégales. L'une, plus grande, abritait le Centre artisanal proprement dit. C'est là que se vendaient les produits de l'artisanat. La deuxième pièce où étaient entreposés les objets d'art collectionnés un peu

62 Boukar Godjé, ancien agent d'entretien du Centre Artisanal de Maroua actuellement en service à la SOCOOPED, Mandara et Hamadou Halilou, agent de la SOCOOPED et fils de l'interprète du Centre Artisanal de Maroua, Guiziga, entretien du 28 janvier 2004 à Maroua.

63 Les noms de ces donateurs du Musée sont jusqu'alors affichés dans le Musée. C'étaient les Lamibé Yaya Dairrou de Maroua, Makaïni de Yagoua, Ahmadou de Doumrou, Golopo de Doukoula et Evélé Oumar sultan de Pouss. 


\section{Autorités coloniales et économie artisanale à Maroua}

partout dans la région servait de Musée d'art local ${ }^{64}$. Les inscriptions "CENTRE ARTISANAL " et " MUSEE D'ART LOCAL » qui furent portées en lettres capitales à l'entrée de ces deux structures existent jusqu'à nos jours. Une tour d'une dizaine de mètres fut élevée au milieu du Centre artisanal, la séparant du Musée. Des escaliers permettant de monter au sommet de cette dernière ont été construits. Un espace sous forme d'une petite cour fut aménagé au-dessus de la tour. ॥ nous a été rapporté que les Blancs vivant à Maroua et bien d'autres qui venaient d'ailleurs aimaient monter là haut et pouvaient ainsi observer la ville de Maroua dans toute sa configuration. Ils y passaient souvent le temps et y prenaient des photographies. De nombreux étrangers venaient de loin s'approvisionner au Centre Artisanal. Aussi achetaient-ils des nappes de table, des poufs, des samaras en cuir, des porte-documents, des porte-feuilles pour ne citer que ces produits-là qu'ils emportaient dans des voitures. Des colis d'objets commandés d'Europe et des autres territoires français d'Afrique étaient expédiés par des vols au départ de Salak où se trouvait un aérodrome. Par la suite, ce bâtiment connut des agrandissements. Compte tenu de l'importance de la production et de la vente des objets artisanaux, l'espace autrefois aménagé devint étroit. Le Centre artisanal connut dès lors une augmentation d'une dizaine de mètres ${ }^{65}$. Jusqu'à aujourd'hui, cet édifice culturel de Maroua existe et c'est là que se vendent les objets artisanaux (fig. 1).

Les Français ne cessèrent de stimuler les artisans. C'est ainsi que certains d'entre eux connurent une mobilité sociale remarquable grâce aux relations privilégiées qu'ils avaient avec les autorités françaises compte tenu de leur talent particulier. Un privilège qui les plaçait à une échelle élevée de la société puisque cela n'était pas donné à n'importe qui et d'ailleurs les gens avaient une perception très particulière des Blancs ${ }^{66}$. Ce fut le cas du maroquinier

64 Boukar Godjé, ancien agent d'entretien du Centre Artisanal de Maroua actuellement en service à la SOCOOPED, Mandara et Hamadou Halilou, agent de la SOCOOPED et fils de l'interprète du Centre Artisanal de Maroua, Guiziga, entretien du 28 janvier 2004 à Maroua.

65 Boukar Godjé, ancien agent d'entretien du Centre Artisanal de Marova actuellement en service à la SOCOOPED, Mandara et Hamadou Halilou, agent de la SOCOOPED et fils de l'interprète du Centre Artisanal de Maroua, Guiziga, entretien du 28 janvier 2004 à Maroua.

66 L'on voudra bien lire certains travaux qui donnent des détails assez importants sur ces personnes qui côtoyaient les Blancs dans l'Afrique coloniale. Amadou Hampaté Bâ, L'étrange destin de Wangrin, Paris, UEG, 1976 ; Mohammadou Bachirou, « Les interprètes sous la période coloniale française dans la région du Nord-Cameroun (1915-1960) "1, mé-
Halilou par ailleurs interprète du Centre artisanal. ॥ reçut la distinction française de "L'Ordre du Mérite artisanal » en ${ }^{195767}$ qui témoigne une fois de plus de l'importance accordée à l'artisanat de Maroua avec des décorations dans ce domaine.

La renommée de ce secteur d'activité de Maroua se diffusa avec sa représentation dans les manifestations culturelles à l'instar des foires et expositions à travers le monde. Bien qu'en 1937, l'artisanat du Cameroun ne fût représenté que par l'art bamoun à l'Exposition internationale de Paris et qu'aucune mention n'eusse été faite de l'artisanat de Maroua, celui-ci fut cependant très présent dans toutes les expositions ultérieures ${ }^{68}$. L'artisanat devint l'une des caractéristiques de la ville de Maroua. A juste titre, l'administrateur-maire de cette cité, Gérard Prestat, écrivait dans un rapport de 1955 que «Maroua est une véritable ville avec une aristocratie fondée sur la naissance, une bourgeoisie commerçante et un artisanat très développé $1{ }^{69}$.

Au sortir de cette partie, l'on constate que par leurs actions nombreuses, les Français réussirent à donner l'orientation qu'ils voulaient à l'artisanat de Maroua. Aussi réussirent-ils à sortir cette activité locale de son style traditionnel d'antan pour l'orienter vers la fabrication de nouveaux objets inspirés des modèles européens. Cette production devait être livrée dans les établissements de vente créés dans ce but. L'artisanat de Maroua changea tant du point de vue technique que commercial, entrant ainsi dans une mouvance de domination ${ }^{70}$. II ne faudrait cependant pas comprendre ces influences françaises de façon isolée, car à travers l'histoire de l'Afrique, les arts du continent en général ont connu des transformations similaires au cours de la période coloniale. Ce qui a poussé certains à parler carrément de la fin des arts traditionnels ${ }^{71}$. La production artistique s'est détournée

moire de maîtrise d'histoire, Université de Ngaoundéré, 1998. 67 Nous avons eu l'opportunité de voir la distinction en question auprès de son fils Hamadou Halilou qui est l'un des informateurs rencontrés à Maroua en 2004.

68 O. Iyébi-Mandjek, op.cit. note 7, p. 33; C. Seignobos et O. Iyébi-Mandjek éd., op.cit. note 3, p. 33.

69 Gérard Prestrat, cité par E. Mohammadou, « Islam et urbanisation $॥$, op. cit. note 3, p. 143.

70 François Wassouni, « L'artisanat africain entre domination et résistance de la période coloniale à nos jours. Le cas de la ville de Maroua au Nord du Cameroun ", Communication présentée à la Conférence du CODESRIA sur Relire l'histoire et l'historiographie de la domination et de la résistance en Afrique, Kampala, Ouganda, 27-29 octobre 2008, à paraître. 71 Frank Willett cité plus haut situe par exemple la fin de l'art traditionnel africain à la fin de la deuxième guerre mondiale qui se situe dans la fourchette des faits relatés dans ce travail. 
de sa vocation et de ses canaux originels pour se banaliser en devenant objet de commerce, un art qui ne servait plus la population locale, mais « un art pour les autres ॥, c'est-à-dire les Européens selon la formule de Joseph Adandé ${ }^{72}$. Les travaux des auteurs comme Jan Vansina, Wole Soyinka, Joseph Adandé et Adedzé Agbenyega sont riches d'analyses relatives à ce sujet73. Parlant justement de ce contexte, Adedzé écrit que « ...Les arts [africains] avaient subi de sérieux changements. Les artistes ne produisaient plus pour des occasions spéciales comme les festivals, mais pour des touristes, surtout Européens et des colons locaux $1{ }^{74}$. Des sociétés coopératives coloniales, des missionnaires et autres Européens spécialisés dans la collecte et le commerce d'objets d'art auraient réalisé des bénéfices durant cette période ${ }^{75}$.

Des années 1930 à 1955, les autorités coloniales françaises s'investirent dans l'organisation du secteur artisanal de Maroua. Des initiatives telles l'introduction

72 Cette expression ressort à plusieurs niveaux dans son article de 2002 cité plus haut.

73 Jan Vansina, «Les arts et la société depuis 1935. Les arts traditionnels 1), dans Ali Mazrui et Christophe Wondji éd., Histoire générale de l'Afrique 1995 [chapitre en ligne] ; Wole Soyinka, "The arts in Africa during the period of colonial rule ", dans General History of Africa, VII : Afrika Under Colonial Domination 1880-1935, Berkeley, University of California Press, 1985, pp. 539-564; Joseph Adandé, op. cit. note 40 ; Agbenyega Adedzé, «L'art africain et l'artiste : perspectives pour le nouveau millénaire 1 , Bulletin du CODESRIA $n^{\text {os }} 3$ \& 4, 2002, pp. 25-29.

74 A. Adedzé, op. cit. note 74, p. 27.

$75 \mathrm{lbid}$. des nouveaux modèles d'objets, l'organisation des filières, l'encouragement des artisans, l'instruction des autorités traditionnelles pour la collecte des objets d'art se développèrent. Dans le contexte d'encadrement de la commercialisation, la SIP ouvre une section artisanale. Dès lors, le commerce des objets artisanaux prend de l'ampleur à Maroua. L'artisanat s'intègre à l'économie moderne selon le vœu des autorités françaises. L'augmentation du volume de la production des objets d'art conduit les administrateurs de l'époque à mettre en place en 1955 le Centre Artisanal. Au-delà de l'objectif commercial, la construction du Centre participe de la promotion de la culture de la région. Dans une ville en plein essor, les étrangers de passage peuvent admirer ce savoir-faire local.

En réalité, il semble que les initiatives françaises ont eu pour objectif de faire de l'artisanat un secteur générateur de revenus pour l'administration coloniale à travers ses coopératives investies dans la vente d'objets artisanaux bien au-delà du territoire camerounais. Le savoir-faire local perdit son originalité d'antan. Pour autant, l'entreprise coloniale aura contribué à poser les jalons d'une politique de promotion de la culture, et même du tourisme. Loin de faire l'apologie de l'administration coloniale française, il faut dire qu'elle a été à la base du développement de l'artisanat, une activité qui fait la spécificité et la renommée de la ville de Maroua jusqu'à aujourd'hui et alimente le marché du tourisme. 


\section{Autorités coloniales et économie artisanale à Maroua}

\section{RéFÉRENCES BIBLIOGRAPHIQUES}

- Joseph ADANDÉ, « L'art africain et l'imaginaire des autres entre le $X V l^{e}$ et le début du XXe siècle. Essai d'analyse diachronique des prémisses d'un processus de " globalisation », Afrika Zamani, ns 9 et 10, 2001-2002, pp. 61-76.

- Agbenyega Adedzé, «L'art africain etl'artiste: perspective pour le nouveau millénaire "), Bulletin du CODESRIA, $n^{\text {os }} 3$ et 4, 2002, pp. 25-29.

- Mohammadou BACHIROU, « Tannage et dynamique socioéconomique à Maroua : 1801-1997 ॥, Rapport de Licence d'histoire, Université de Ngaoundéré, 1997.

- Ghislaine DéGAtier et Olivier Iyébi-MandJek, « L'évolution de l'artisanat du cuir à Maroua », Rapport multigraphié, AFVP, 1992.

- Hans DominIK, "Expédition et combat contre Maroua ", TA-49, 1902, document consultable aux Archives Nationales de Yaoundé.

- Étienne FÉAu, « L'art africain dans les collections publiques européennes I), in Caroline Gaulthier-Kurhan dir., Le patrimoine culturel africain, Paris, Maisonneuve et Larose, 2001, pp. 229-295.

- Henri Fréchou, L'élevage et le commerce du bétail dans le Nord-Cameroun, Cahiers de l'ORSTOM, Série Sciences Humaines, Vol. III, n² 2, 1966.

-Aïssatou-Boussoura Garga, «Les mutations commerciales dans la région de Maroua pendant la première moitié du XXe siècle "I, Mémoire de Maîtrise d'histoire, Université de Yaoundé I, 2000.

- Jacques GIRI, Histoire économique du Sahel, Paris, Karthala, 1994.

- Abdouraman Halirou, «Yaya Daïrou, Lamido de Maroua (1943-1958) II, Mémoire de Maîtrise d'Histoire, Université de Ngaoundéré, 1997.

- Amadou Hampaté BÂ, L'étrange destin de Wangrin, Paris, UEG, 1976.

- Jacques Kernache, Jean-Louis Paudrat et al., L'art africain, Paris, Mazenod, 1988.

- Olivier IyÉBI-MANDJEK, « L'artisanat du cuir à Maroua : étude de marché ॥, Rapport multigraphié, 1993.

- avec Nicole MAINET « L'artisanat d'art à Maroua (NordCameroun) ") dans Annales de la Faculté des Lettres et Sciences Humaines, n 8, Yaoundé, 1977, pp. 159-186.

- Eldridge Mohammadou, L'Histoire des Peuls Férôbé du Diamaré : Maroua et de Pétté, Tokyo, ILCAA, 1976.

- « Islam et urbanisation dans le Soudan Central au XIX siècle. La cité de Maroua (Nord-Cameroun) "), dans The Proceedings of international conference on urbanism in Islam (ICUIT), Tokyo, 22-28 octobre 1989, pp. 117-154.

- « L'empreinte du Borno sur les Foulbé de l'Adamawa et leur langue ॥, dans Ngaoundéré-Anthropos, Revue de sciences sociales, Vol. I, Yaoundé, Imprimerie Saint-Paul, 1996, pp. 71-90.
- « Les interprètes sous la période coloniale française dans la région du Nord-Cameroun (1915-1960), Mémoire de Maîtrise d'histoire, Université de Ngaoundéré, 1998.

- Les Lamidats du Diamaré et du Mayo-Louti au XIXe siècle, Tokyo, ILCAA, 1988.

- Engelbert Mveng, Histoire du Cameroun, Paris, Présence Africaine, 1963.

- Vincent NéGRI, « La création du droit du patrimoine culturel en Afrique "), dans Caroline Gaulthier-Kurhan (dir.), Le patrimoine culturel africain, Paris, Maisonneuve et Larose, 2001, pp. 321-340.

- Mahamat PABA SALÉ, « Maroua : aspects de la croissance d'une ville du Nord-Cameroun ") (Des années 50 à nos jours), thèse de doctorat de $3^{e}$ cycle en Géographie, Université de Bordeaux III, 1980.

- Marcel RoupsARD, Nord-Cameroun. Ouverture et Développement., Coutances, Claude Bellée, 1987.

- Christian Selgnobos, « De l'objet culturel au produit d'artisanat, de l'influence du tourisme au NordCameroun "I, Enjeux, Publication de la FPAE, Yaoundé, $n^{\circ}$ 5, 2006, pp. 30-35.

- avec Olivier lyéBl-MANDJECK éd., Atlas de la province de L'Extrême-Nord, Paris, IRD, 2000.

- Magloire SOMÉ, « Les cultures africaines à l'épreuve de la colonisation ॥, Afrika Zamani, nºs 9 et 10, 2001-2002, pp. 41-58. - Wole SOYINKA, « The arts in Africa during the period of Colonial Rule ॥, dans General history of Africa, VII : Africa under colonial domination 1880-1935, Berkeley, University of California Press, 1985, pp. 539-564.

- Jan VANSINA, « Les arts et la société depuis 1935. Les arts traditionnels ") dans Ali Mazrui et Christophe Wondji dir., Histoire générale de l'Afrique, 1995 [chapitre en ligne].

- François WASSOUNI, « L'artisanat africain entre domination et résistance de la période coloniale à nos jours. Le cas de la ville de Maroua au Nord du Cameroun ", Communication présentée à la Conférence du CODESRIA sur Relire I'histoire et I'historiographie de la domination et de la résistance en Afrique, Kampala, Ouganda, 27-29 octobre 2008 (à paraître).

- « L'artisanat dans l'Extrême-Nord du Cameroun : XIXe-XXe siècles $\|$, Mémoire de DEA d'histoire, Université de Ngaoundéré, 2004.

- «Production, consommation et commercialisation du cuir à Maroua : XIX $\mathrm{X}^{\mathrm{e}} \mathrm{XX} \mathrm{X}^{\mathrm{e}}$ siècles ॥, Mémoire de Maîtrise d'histoire, Université de Ngaoundéré, 2002.

- Frank Willett, L'art africain, Paris, Éditions Thames\&Hudson, traduction française, 1990.

- Oscar Zimmerman, Durch busch und steppe, von Campo bis zum Schari 1892-1902, Berlin, 1909. 